\title{
INFINITE MULTIPLETS*
}

\author{
Yoichiro Nambu
}

The Enrico Fermi Institute for Nuclear Studies and Department of Physics

The University of Chicago, Chicago, Illinois

\section{LEGAL NOTICE}

This report ans prepared as an account of Government sponsored work. Neither the United This repor sas com any person acting on behall of the Commission:

States, nor the Cocnmission, hor any person action, expressed or implied, with respect to the accuA. Makes any warranty or representation, expressed or implied, with respect the the use racy, completeness. Or usefulaess or the intors disclosed in this report may not thfringe of any information, appar

primately ouned rights: or B. Assumes any liabilities with respect to the use of, or for damnges result use of any information. apparatus, method, or process disclosed in this report. As used in the above, "person acting on behalf of the Commission" includes any employe or contractor of the Commission, or employee of such contractor, to the extent that surb employee or conides access to, any information pursuant wo his employment or contract with the Commissios, or his employment with such contractor.

(Talk presented at the International Theoretical Physics Conference on Particles and Fields, Rochester, 1967)

* Supported in part by the U. S. Atomic Energy Commission. 


\section{DISCLAIMER}

This report was prepared as an account of work sponsored by an agency of the United States Government. Neither the United States Government nor any agency Thereof, nor any of their employees, makes any warranty, express or implied, or assumes any legal liability or responsibility for the accuracy, completeness, or usefulness of any information, apparatus, product, or process disclosed, or represents that its use would not infringe privately owned rights. Reference herein to any specific commercial product, process, or service by trade name, trademark, manufacturer, or otherwise does not necessarily constitute or imply its endorsement, recommendation, or favoring by the United States Government or any agency thereof. The views and opinions of authors expressed herein do not necessarily state or reflect those of the United States Government or any agency thereof. 


\section{DISCLAIMER}

Portions of this document may be illegible in electronic image products. Images are produced from the best available original document. 
$\underline{C} \underline{O} \underline{N} \underline{T} \underline{E} \underline{N} \underline{T} \underline{S}$

I. INTRODUCTION -

II. CHOICE OF THE DYNAMICAL GROUP G AND ITS REPRESENTATION -.... 5

III. MODELS BASED ON SL $(2, c)$

1) Form factors - 8

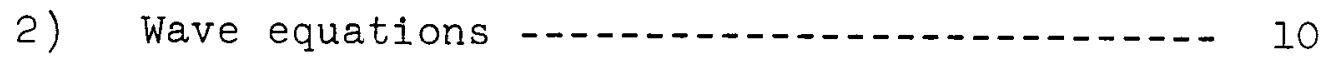

3) Minimal electromagnetic interaction -...- 13

IV. MODELS BASED ON SO $(4,2) \sim \operatorname{SU}(2,2) \ldots \ldots \ldots \ldots$

V. MODELS BASED ON SU $(3,1)$

VI. PROBLEMS OF QUANTIZED FIELDS -........... 19

VII. SCATTERING PROCESSES -

VIII. APPLICATION TO HADRON PHYSICS -...........- 25

References - 


\section{INFINITE MULTIPLETS}

\section{INTRODUCTION}

The main ingredients of the method of infinite multiplets consist of :

1) the use of wave functions with an infinite number of components for describing an infinite tower of discrete states of an isolated system (such as an atom, a nucleus, or a hadron),

2) the use of group theory, instead of dynamical considerations, in determining the properties of the wave functions.

The group theory is used in three ways. It determines the relativistic transformation properties of the wave function as an infinite-dimensional (not necessarily irreducible) representation of the Lorentz group. It determines the internal quantum numbers and degeneracy structure of the mass levels as a consequence of an assumed symmetry grcup in the rest frame of the particle (i.e., a little group). It is also used to define various observables. The smallest of the symmetry group is the rotation groupSO(3), which gives rise to the $(2 j+1)$ - fold degeneracy of states witr spin $j$. In general, we can have a larger degree of degeneracy, including that due to the so-called internal symmetry like $\mathrm{SU}(2)$, SU(3), etc。 Thus we are led to a hierarchy of groups 


$$
\begin{aligned}
& S_{0} \subset S \sim L \subset P \\
& \cap \cap \\
& G_{0} \subset G
\end{aligned}
$$

Here $S_{O}=S O(3)$, and $G_{O}$ is the degeneracy group (dynamical symmetry group). S (the internal Lorentz group) and $G$ are their Lorentz closures obtained by boosting them. Usually $G_{O}$ is compact and semi-simple. $G_{O}$ may be just a direct product $G_{O}=S_{O} \otimes A$, where $A$ is an internal symmetry group, and hence $\mathrm{G}=\mathrm{S} \otimes \mathrm{A}$. There are, however, other non-trivial examples, like the hydrogen atom, the isotropic harmonic oscillator, or the SU(5) model of hadrons, in which $G$ is not such a direct product. We take an (infinite dimensional) representation of the non-compact group $\mathrm{G}$ and consider it, in addition, as a function of the space-time coordinates; thus making it an infinite component field $\psi_{n}(x)$. The discrete index $n$ arises because reduce $\psi$ with respect to the compact subgroup $G_{0}$. In general, we would like to choose $G$ and $\psi$ in such a way that the set $\left\{{ }_{n}\right\}$ coincides with the complete set of actual physical levels. However, sometimes it may be more convenient to relax this condition. The energy-momentum 4-vector and the total angular momentum (or the physical Lorentz group L) can be defined on this field, and $\left\{\psi_{n}(x)\right\}$ becomes also a representation of the Poincaré group (inhomogeneous Lorentz group) P. However, we are not trying to combine $\mathrm{P}$ and $\mathrm{G}$ into a larger group, so we dismiss 
herewith all the problems related to the theorems of McGlinn, o'Rafaertaigh, etc.

(As was mentioned already, the group $G$ and its Lie algebra serve the purposes of defining the spin and other quantum numbers, ensuring relativistic covariance, and expressing physical observables such as the mass and currents. In this sense $G$ is sometimes called a dynamical group ${ }^{2}$ or a noninvariance group ${ }^{3}$, and its Lie algebra forms a spectrum generating algebra and a transition operator algebra ${ }^{4}$ as well as a symmetry algebra.)

The substitution of dynamics with group theory has been one of the recent trends in elementary particle physics. Its motivation comes from the fact that, having seen a general qualitative success of the quark model picture of hadrons, one wants a more quantitative, but simple and unified description of hadron phenomena without assuming detailed dynamical mechanisms and even the existence of the yet unknown quarks as real physical particles. In this kind of approach, the wave function $\psi\left(x_{1}, x_{2}\right)$ for a two-particle bound state, e.g., a meson as a quark-antiquark system, can be brought into a more abstract form (departing from a space-time description of the internal structure) by expanding it with respect to an appropriate discrete basic $\left\{f_{n}\right\}$ :

$$
\begin{aligned}
& \psi\left(x_{1}, x_{2}\right) \rightarrow \sum^{\psi}(x, r) \\
&-\sum_{n} C_{n}(x) f_{n}(r) \\
& x=\left(x_{1}+x_{2}\right) / 2, \quad r=x_{1}-x_{2}
\end{aligned}
$$


The set $\left\{C_{n} \psi(X)\right\} \equiv\left\{\Psi_{n}(X)\right\}$ then defines an infinite component field. Thus the emergence of infinite component fields seems entirely natural from this viewpoint.

There are other considerations that have led some people to turn their attention to infinite component fields. For example, the scattering amplitudes cannot be made well behaved. (i.e., to satisfy superconvergent relations and sum rules) unless one introduces an infinite tower of states; whereas, vertex functions acquire damping form factors if the field. transforms as a unitary (and therefore infinite-dimensional) representation of the Lorentz group. I would like to remark, however, that the unitarity requirement in quantum mechanics for the probability density does not, in itself, lead to the necessity of having a unitarity representation of $G$. As we know well, it is the unitary representation of the Poincaré group $P$ that we need, not necessarily of $L$ or $G$. (In order to secure the unitarity with respect to $P$, however, it will be necessary to invoke wave equations.)

Starting from more abstract principles, one can also arrive at the infinite component fields. Thus Takabayashi's long series of work ${ }^{5}$ has been motivated by the non-local (bilocal, and later, quadrilocal) theory of Yukawa ${ }^{6}$. The pioneering work of Majorana ${ }^{7}$ was motivated by the non-existence (at that time) of anti-particles; a fact which is not valid any longer. 
Because of the different viewpoints from which different people have started, there seems to be no consensus about the position of the infinite component fields relative to the conventional mathematical tools in elementary particle physics. One may use them, for example, as part of the S-matrix theory in defining an infinite multiplet of asymptotic fields, or simply as a basis for the expansion of an amplitude. Or one may regard them as a new possibility within the framework of axiomatic field theory. One may also consider them as belonging to Lagrangian field theory. In the last circumstance, a field will be subject to a wave equation which will determine the mass spectrum of a system, as well as its interaction with external fields, etc. The question is whether one can make a formulation which is free of internal inconsistencies, and besides, is useful and relevant to the actual physics of hadrons. However, the more we demand out of it, the more problems we must also face. This will be seen in the following.

\section{CHOICE OF THE DYNAMICAL GROUP G AND ITS REPRESENTATION}

The first contact between mathematics and physics is made when one decides on the choice of the group $G$ and its representation. For this purpose, the following considerations are in order:

1) The degeneracy structure (exact or approximate) of levels, which will depend on the symmetry group $G_{0}$. 
2) The nature of low lying excitations; i.e., whether there exist rotational excitations, radical excitations, etc.

3) The behavior of form factors and transition amplitudes between levels.

For example, if radial excitations in a two-body system are frozen, we have for $G_{0}$ the smallest possible one $G_{0}=S O(3)$ ignoring intrinsic spin and other internal degrees of the constituents, and $G=S O(3,1)$. We should then consider an irreducible representation of $\mathrm{SO}(3,1) \sim \mathrm{SL}(2, \mathrm{C})$, or a finite sum of IR's in order to accommodate parity and other additional operators. Since an IR contains, when reduced with respect to the rotation group, different spin values $j$ (either integer or half-integer) only once for each $j$, we get a single Regge trajectory (combining both signatures).

When radial excitations are included and the degeneracy group $G_{0}$ is larger than $S O(3)$, we run into two conspicuous physical examples that have been extensively studied.

1) $G_{O}=\mathrm{SO}(4), G=\mathrm{SO}(4,1)$ or $\mathrm{SO}(4,2) \sim \mathrm{SU}(2,2)$, (hydrogen atom).

2) $G_{0}=\mathrm{SU}(3), G=\mathrm{SU}(3,1)$ or $U(3,1)$, (harmonic oscillator). The number of times an IR of the subgroup $G_{0}$ occurs in an IR of $G$ depends on the representation, so we have to choose a particular kind (usually a degenerate representation) that 
corresponds to the actual multiplet structure of levels. When reduced with respect to the rotation group, it gives rise to an infinite recurrence of Regge trajectories, but each trajectory is not simply related to the reduction with respect to the internal Lorentz group $S \subset G$.

III. MODELS BASED ON SL $(2, c)$

Since $\mathrm{SO}(3,1) \sim \mathrm{SL}(2, \mathrm{c})$ is the minimum group in our approach, we will take this as a basic example to discuss some characteristic problems. A finite-dimensional $\operatorname{IR~} D\left(j_{1}, j_{2}\right)$ or $\operatorname{SL}(2, c)$ is given in terms of a symmetric tensor product

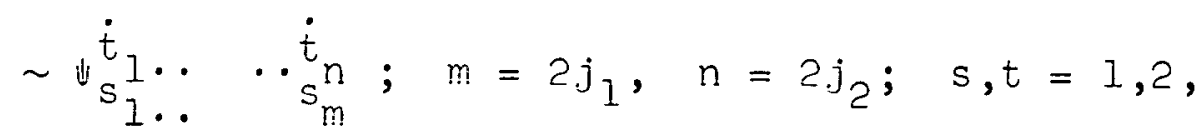

built out of basic spinors

$$
\psi_{S} \sim D\left(\frac{1}{2}, 0\right), \quad \|^{\dot{t}} \sim D\left(0, \frac{1}{2}\right),
$$

with the property

$$
D\left(j_{1}, j_{2}\right) \approx D\left(j_{2}, j_{1}\right)^{*} .
$$

It decomposes into the spin substates as

$$
\left.D\left(\left|j_{1}-j_{2}\right|\right) \oplus D\left(\left|j_{1}-j_{2}\right|\right)+1\right) \oplus-\cdots \oplus D\left(\left|j_{1}+j_{2}\right|\right) \text {. }
$$

The general IR of $\operatorname{SL}(2, c)$ can be obtained from this by analytic continuation, fixing $k \equiv j_{1}-j_{2}$, and letting $j_{1}+j_{2}+1 \equiv c$ take an arbitrary complex value. Thus we write an IR as $D(k, c)$, which will decompose in general as $\sum_{n=0^{\oplus}}^{\infty} D(|k|+n)$. It also turns out that $D(k, c)=D(-k,-c)$. 
The space reflection (parity) may be defined on $D\left(j_{1}, j_{2}\right)$ as $j_{1} \rightarrow j_{2}$, which means $D(k, c)-D(-k, c)=D(k,-c)$.

Let us write the six infinitesimal generators as

$$
i L_{i}=\epsilon_{i j k} \quad \Lambda_{j k}, \quad i K_{i}=\Lambda_{i o} \cdot
$$

$\underset{\sim}{L}$ operates within each subspace $D(j) ; \underset{\sim}{K}$ has matrix elements between neighboring $\mathrm{D}(j)$ 's. Different representations differ in the matrix elements of $\mathrm{K}$.

An IR $D(k, c)$ is unitary; i.e., $K$ is Hermitian, if and only if

a) $c=$ imaginary (principal series)

or

b) $\mathrm{k}=0,0<\mathrm{c}<1 \quad$ (supplementary series)

Since $k$ is the lowest spin value that occurs in an IR, it is fixed by physical considerations. Thus $c$ is the only variable parameter.

1) Form factors

Define a local scalar product $\sum_{n} \bar{\Psi}_{n}(x) \bar{\Psi}_{n}(x)$ where $\left\{\bar{\Psi}_{n}\right\}$ transforms as $D(k, c)$ under $S$. In a unitary representation, $\psi^{*}=\psi^{*}$. In general, we have to use an adjoint field

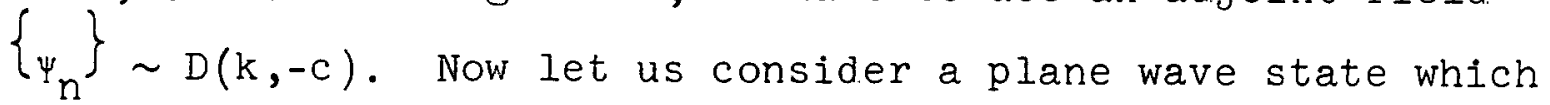
is at rest and has definite spin values $j$ and $j_{z}$. A moving state can be obtained from this by applying the Lorentz transformation $L$ to both spin and orbital parts (boosting). In this way we can define scalar vertex function for a transition between two Fourier components (momenta) $\mathrm{p}_{\mu}$ and $\mathrm{p}_{\mu}^{\prime}$ having 
spin and helicity $(j, h)$ and $\left.j^{\prime}, h^{\prime}\right)$, respectively. Essentially it is given by the matrix element

$$
<j^{\prime}, h^{\prime}\left|\exp \left[i \vartheta K_{z}\right]\right| j, h>
$$

between two spin substates of $\psi_{n}$ and $\bar{\psi}_{n}$, where tanh $\vartheta=v$ is the relative velocity between the two states. We have the simple relation

$$
\gamma \equiv \cosh \vartheta=p \cdot p^{\prime} / m^{\prime}=1+\frac{(m-m)^{2}-t}{2 m m^{\prime}}
$$

For the finite-dimensional representation, the t-dependence (form factor) of ( $I$ ) is essentially like a polynomial, but we obtain non-trivial results in the infinite-dimensional case. For example, with $j=j^{\prime}=0$,

$$
<0\left|\exp \left[i \vartheta \mathrm{K}_{z}\right]\right| 0>=\sinh (\mathrm{c} \vartheta) / \mathrm{c} \sinh \vartheta \text {. (3) }
$$

We make the following remarks:

a) The maximum degree of growth of (1) for large $\gamma \sim-t / 2 m^{2}$ is like $\lesssim \gamma^{j_{1}}+j_{2}=\gamma^{c-1}$ in the sense that there exists at least one matrix element for any fixed $j$ and $j^{\prime}$ which behaves like $\sim \gamma^{c-1}$. (This follows from elementary considerations about the behavior of a finite-dimensional $D\left(j_{1}, j_{2}\right)$, which can be analytically continued.) Thus for unitary representations the matrix element $\rightarrow 0$ as $\gamma \rightarrow \infty$, but it does so only like $\sim \gamma^{-1}$ asymptotically. It should be mentioned that this applies to the overall matrix element, and not to the invariant form factors defined after separating out kinematic factors. Similar arguments can be made for vector (current) and other vertices when these can be defined. 
b) The matrix elements (1) have the right threshold behavior ${ }^{9}$. This can be understood from the fact that the Lorentz generator $K$ has a selection rule $\left|j-j^{\prime}\right| \leqslant 1$, so that the matrix element must behave like $\sim \vartheta^{\left|j-j^{\prime}\right|} \sim v^{\left|j-j^{\prime}\right|}$.

c) Analyticity and crossing. The matrix elements (1) for infinite representations have a branch point at $\gamma=-1$, corresponding to the normal threshold $t_{0}=\left(m+m^{\prime}\right)^{2}$, and at $\gamma=\infty$. This is due to the fact that the imaginary Lorentz transformation $\gamma=\cosh \vartheta_{\pi}=-1$, which is a compact rotation, acts on an infinite basis. For the same reason, the vertices do not have crossing symmetry ${ }^{10}$ : the transition element between positive $\left(p_{0}>0\right)$ and negative $\left(p_{0}^{\prime}<0\right)$ Fourier components as calculated from Eq. (1) is not the same as the usual analytic continuation of the positive-positive matrix element. In fact, the former should be regular at the threshold $(\gamma=-1)$, and singular at $\gamma=1$. The two form factors can be related only by analytic continuation round $\infty$. These points can be explicitly seen from Eq. (3).

\section{2) Wave equations}

Most wave equations that have been considered are Iimited to quadratic or Iinear (Majorana ${ }^{7}$-Gelfand-Yaglom ${ }^{11}$ type) differential equations, if only for reasons of mathematical simplicity and nothing else. These equations will be of the form 


$$
\left(p_{\mu} p^{\mu}+\alpha w_{\mu} w^{\mu}-\beta\right) \psi=0
$$

or

$$
\left(r_{\mu} p^{\mu}-x\right) \Psi=0
$$

Where $w_{\mu}$ is the Pauli-Lubánski vector, $\Psi$ is in general reducible under $\operatorname{SL}(2, c)$, and $\alpha, \beta, x$ may be functions of the Casimir operators. In fact, we cannot take a single arbitrary IR for $\Psi$ in general. To define parity, we need a pair $(k, c)$ and $(k,-c)$. In order to define a current, we need also their adjoints. Finally, in the case of linear equations, we need a pair of IR's $(k, c)$ and $\left.k^{\prime}, c^{\prime}\right)=(k \pm l, c)$ or $(k, c \pm l)$ in order to realize current operators $T_{\mu} 11,16$. Thus we end up w1th up to eight IR's. The Majorana equation $(k, c)=\left(0, \frac{1}{2}\right)$ or $\left.\left(\frac{1}{2}, 0\right)\right)$ is the only case where a single IR suffices.

These equations, as they may look simple and elegant, suffer from well-known deficiencies from the physical point of view:

a) The mass spectrum $m(J) \rightarrow 0$ as the spin $J \rightarrow \infty l l$. (Exceptions to this are the cases, with Eq. (3a), i) $\alpha=0$, $\mathrm{m}^{2}=$ const., and ii) $\beta=0, J(J+1)=\alpha, \mathrm{m}^{2}$ arbitrary.)

b) There is also a continuous family of lightlike solutions, and even worse, of unphysical spacelike solutions as well $1^{7,12,13}$. (There have been some proposals ${ }^{14,15}$ to interpret the spacelike solutions in physical terms.) 
C) In the case of Majorana representation, the massive and massless states come in with only one sign of energy (or frequency). Thus a particle is not accompanied. by an anti-particle. If, however, a pair of IR's are used in Eq. (3b), we can have both signs. So this is not a general feature of infinite-dimensional representations.

The above remarks do not necessarily apply to more complicated (non-Gelfand-Yaglom type) equations; e.g., when $x$ in $E q .(3 b)$ is a function of $p^{2}$. We can get a rising spectrum $m \propto J$ by taking $k \alpha p^{2}$. But the other difficulties still persist.

Another way is to take an infinite (or finite) set of IR's $\mathrm{D}(\mathrm{k}, \mathrm{c}), \mathrm{k}=0,1,2, \ldots$ (Boson) or $1 / 2,3 / 2,--$ (Fermion) with fixed $c$, and postulate

$$
\begin{aligned}
& {\left[p^{2}-f(k)\right] \psi=0} \\
& {\left[w^{2}-g(k)\right] \psi=0}
\end{aligned}
$$

in such a way that only certain spin values are selected for each $k$. In this case the space $\{y\}$ is much larger than is necessary to accommodate physical levels, but it has the advantage of being free of all unphysical solutions. For example, we obtain a rising linear spectrum $m^{2}=a j+b$ by choosing

$$
f(k)=a k+b, \quad g(k)=-f(k) k(k+1) .
$$

Eq. (3c) can be derived from a Lagrangian by introducing an auxiliary field. 
3) Minimal electromagnetic interaction

We discuss here briefly the problem of electromagnetic interaction, especially the magnetic moment. It is indeed an interesting feature of our approach that non-trivial electromagnetic properties can result even with the assumption of minimal electromagnetic interaction. This assumption is reasonable at least if we are effectively dealing with a composite system in which only one of the constituents, considered elementary, is charged.

The current is uniquely defined as

$$
\begin{aligned}
& j_{\mu}(x)=\bar{\Psi}(x) V_{\mu} \psi(x), V_{\mu}=\delta I / \delta p^{\mu}, \\
& \partial_{\mu} j_{\mu}(x)=0
\end{aligned}
$$

if the free Lagrangian density is $\bar{\psi} L \psi$. In addition to the form factors discussed above, we will also obtain non-trivial static magnetic moment (and higher moments) by taking the matrix element of $j_{\mu}$. The result is, however, again rather unphysical in the case of the already unphysical equations (3): the $g$ factor is $=0$ for Eq. (3a), and < 0 for Eq. (3c) if only a pair of IR's are used ${ }^{16}$. For non-Gelfand-Yaglom type equations, the situation can be different.

IV. MODELS BASED ON SO $(4,2) \sim \operatorname{SU}(2,2)$

As has been discovered by various authors ${ }^{17-19}$, the group $S O(4,2) \sim S U(2,2)$, rather than $S O(4,1)$, provides a nice framework in which to formulate the problem of the hydrogen 
atom. It is also interesting that $\mathrm{SU}(2,2)$ is the group generated by Dirac matrices, and therefore, it serves as a prototype of the theories like $\mathrm{SU}(6,6)$. But this connection is probably accidental.

SO $(4,2)$ contains $S O(4) \otimes S O(2)$ as its compact subgroup, so that there are three discrete quantum numbers to label a state. Since the actual hydrogen atom has only two, corresponding to so(4) degeneracy, we need select a special degenerate representation in which the third quantum number is not Independent of the other two. In fact, we find a unique representation that serves our purpose.

We label the six fictitious dimensions $0,1,2,--5$, with metric +----+. The physical space is identified with 1,2,3. The secret of success in reformulating the non-relativistic hydrogen atom lies in the fact that, using the physical space as the carrier space, the 15 generators $M_{\alpha \beta}$ of $S C(4,2)$ may be identified as

$$
\begin{aligned}
& M_{45}+M_{50}=r=\left(r^{2}\right) 1 / 2 \\
& M_{45}-M_{50}=r \Delta \\
& M_{41}-M_{01}=x_{1} \\
& M_{01}+M_{41}=x_{i} \Delta+2 x_{k} \partial^{k} \partial_{i}+2 \partial_{i} \\
& M_{51}=i r \partial_{i} \\
& M_{41}=i\left(x_{i} \partial^{i}+1\right)
\end{aligned}
$$

i 
These operators are Hermitian, and hence give a unitary representation, if the metric is defined by

$$
(\psi, \psi)=\int \psi *(\underline{r}) \frac{1}{r} \psi(\underline{r}) d^{3} x
$$

Now multiply the schrbdinger equation

$$
(H+B) \psi=0, \quad H=\frac{1}{2} \Delta-\frac{\alpha}{r}
$$

by $r$, and use Eq. (4). We get immediately

$$
\left[\left(\frac{1}{2}+B\right) M_{50}+\left(\frac{1}{2}-B\right) M_{54}-\alpha\right] \psi=0
$$

Once this is achieved, we can also go to a discrete basis $\psi(\underset{m}{r}) \rightarrow \psi_{n}$ using the quantum numbers of the compact subgroup.

Eq. (7) can be solved by making a hyperbolic rotation in the (04) plane:

$$
\psi=\exp \left[i \Theta M_{O 4}\right] \|^{\prime}, \tanh \theta=\left(\frac{1}{2}-B\right) /\left(\frac{1}{2}+B\right) \text {, if } B>0 .(8)
$$

The transformed equation

$$
\left\{\left[\left(\frac{1}{2}+B\right)^{2}-\left(\frac{1}{2}-B\right)^{2}\right]^{1 / 2} M_{50}-a\right\} \psi^{\prime}=0
$$

immediately gives the familiar spectrum

$$
\sqrt{2 B} M_{50}=\alpha \text {, or } B=\alpha^{2} / 2 M_{50}
$$

where the discrete "principal quantum number" $M_{50}=n$ takes the values $1,2,3, \ldots$ in this representation. Similarly, the ionized states $B<0$ follow by rotating into the direction 4: $B=-\alpha^{2} / 2 M_{54}$, where the non-compact generator $M_{54}$ now takes continuous values.

It was shown by Fronsdal ${ }^{19}$ that the wave function $\Psi_{n}$ can be made into a field ${ }_{n}(x)$ with a Galilei invariant 
Hamiltonian. The electromagnetic interaction may be introduced according to the rule $\underset{m}{P}-\underbrace{P}-e \underset{m}{A}(\underbrace{x}_{m})$ The equation is still linear in the $\mathrm{SO}(4,2)$ generators. This corresponds to a description of the hydrogen atom in which

$$
\mathrm{M}_{\mathrm{p}}>\mathrm{M}_{e}, \underset{m}{\mathrm{X}}=\underline{m}_{e}, \underset{m}{r}=\underline{m p}_{\mathrm{p}}-\underline{r}_{e} \text {. }
$$

The peculiar feature of this problem is the rotation (8), first noted by Barut ${ }^{17}$. Since $\theta$ is different for different levels, the eigenfunctions ${ }_{B}$ are not orthogonal; they are orthogonal only with respect to a metric operator $M_{50}-M_{54}$. This nicely solves the old dilemma that physical transition operators cannot be represented by generators since the latter induce transitions only between neighboring orthogonal states in a representation.

So much for the non-relativistic hydrogen atom. By assigning the subspace (0123) to the Minkowski space, one can formally write down linear relativistic wave equations with so(4) degeneracy. But again we tend to run into the difficulties encountered in the $\mathrm{SL}(2, \mathrm{c})$ case. Nevertheless, some model equations without spacelike solutions have been found ${ }^{18,19}$. As an example, we mention

$$
\begin{aligned}
& {\left[\Gamma_{\mu} \mathrm{p}^{\mu}+\frac{1}{\mathrm{k}} \mathrm{SE} \mathrm{k}_{\mu} \mathrm{p}^{\mu}-\alpha \gamma_{\mu} \mathrm{p}^{\mu}\right] \quad v^{\prime}=0} \\
& I_{\mu}=\mathrm{M}_{5 \mu}, \quad \mathrm{S}=\mathrm{M}_{54}
\end{aligned}
$$

$w$ is a direct product of Dirac and SO $(4,2)$ representations. This equation has a discrete hydrogen-like mass spectrum 
$m= \pm x \sqrt{1-\alpha^{2} / n^{2}}$, a continuous spectrum $|m|>x$, and a family of massless solutions, but is free of spacelike solutions.

\section{MODELS BASED ON SU $(3,1)$}

This group is the minimum Lorentz extension of SU(3), the degeneracy group of the 3-dimensional isotropic harmonic oscillator. Perhaps the harmonic oscillator is an appealing idealization of the orbital motion in the quark model of hadrons as well as the nuclear shell model. At the same time, it has an advantage in the simplicity of mathematical properties.

In order to construct a representation, we introduce the 4-dimensional oscillator variables $a_{\mu}, a_{\mu}^{+},(\mu=0-3)$ with $\left[a_{\mu}^{+}, a_{v}\right]_{-}=G_{\mu v}$ (metric +---). (Annihilation operators are thus $a_{1}, a_{2}, a_{3}$ and $a_{0}{ }^{+}$.) Then the 16 combinations $a_{\mu}{ }^{+} a_{u}$ form generators of $U(3,1)$. The Lorentz rotation group $O(3,1)$ is generated by the antisymmetric tensor $a_{\mu}^{+} a_{v}-a_{v}^{+} a_{\mu}=i L_{\mu v}$ whereas the symmetric tensor $i\left(a_{\mu}^{+} a_{v}+a_{v}^{+} a_{\mu}\right)=i \Gamma_{\mu v}$ corresponds to deformation and dilatation. The SU(3) subgroup $\left(G_{0}\right)$ is generated by $a_{i}, a_{i}^{+}(i=1,2,3)$ alone.

In order to describe the harmonic oscillator, then, we have to suppress the fourth degree of freedom. This can be done by going to $\mathrm{SU}(3,1)^{20-22}$. We impose the condition

$$
-a_{\mu}^{+} a^{\mu}=\sum_{i=1}^{3} a_{i}^{+} a_{i}-a_{0}^{+} a_{0}=\lambda
$$


which relates $a_{0}^{+} a_{0}$ to $N=\sum a_{i}^{+} a_{i}$. It can be shown that the substitution $a_{0} \rightarrow \sqrt{\mathrm{N}-\lambda}, a_{0}^{+} \rightarrow \sqrt{\mathrm{N}-\lambda}$ in the $\mathrm{U}(3,1)$ generators leaves their commutation relations unchanged except that now $\Gamma_{\mu}^{\mu}=-2 \lambda$. We get following $I R^{\prime} s$

$$
\begin{aligned}
\text { i) } \lambda & \leqslant 0 \text {, unitary. } \\
\text { ii) } \lambda & =\text { integer }>0, N \leqslant \lambda \text {, finite non-unitary. } \\
\text { iii) } \lambda & =\text { integer } \geqslant 0, N>\lambda \text {, discrete unitary. }
\end{aligned}
$$

The behavior of form factors according to these representations is $\sim \gamma^{\lambda}$ for $\left.i\right)$ and $\left.i i\right)$, and $\sim \gamma^{-1-\lambda}$ for iii). They have an anomalous singularity at $\gamma=0$ or $t=\mathrm{M}^{2}+\mathrm{M}^{\prime 2}$, the physical meaning of which is unclear. (It may be a little surprising that we do not obtain the gaussian form factor of the harmonic oscillator, but the latter follows as a non-relativistic limit in which $|\lambda| \rightarrow \infty^{2 l}$. At any rate, by a suitable choice of $\lambda$ we can get any asymptotic power behavior.)

The wave equations based on $\mathrm{SU}(3,1)$ are necessarily quadratic, since $\Gamma_{\mu \nu}$ is the only available tensor. Thus a typical form will be

$$
\left[\Gamma_{\mu U} p_{\mu} p^{U}-\alpha p_{\mu} p^{\mu}+\beta\right] \psi=0
$$

with the mass spectrum

$$
\mathrm{m}^{2}=\beta /(\alpha+\lambda-\mathrm{N}), \mathrm{N}=0,1,2,-
$$

This is physical only if $\beta>0, \alpha+\lambda-N>0$. There are no other solutions corresponding to a real four-vector $P_{\mu}$. If we 
take a fourth order equation, we can also obtain an infinity of levels with a hydrogen-like accumulation point.

Another form similar to Eq. $(3 \mathrm{C})$ is based on $U(3,1)$, and eliminates the unphysical solutions by means of a supplementary condition 22,23 . We take

$$
\begin{aligned}
& {\left[p_{\mu} p^{\mu}+x^{2}\left(a_{\mu}^{+} a^{\mu}-c\right)\right] \Psi=0,} \\
& \left(p_{\mu} a^{+\mu}\right) \psi=0\left[\text { or }\left(p_{\mu} a^{\mu}\right)\left(p_{u} a^{u^{+}}\right) \psi=0\right]
\end{aligned}
$$

The mass spectrum is strictly linear

$$
M^{2}=x^{2}(N+C) \text {, }
$$

whereas the supplementary condition suppresses the fourth degree of freedom: $a_{0}^{+} \psi=0$ for time-like solutions, and eliminates spacelike solutions completely: $a_{i}^{+} \psi=0 \rightarrow \psi=0$. In this scheme, $\lambda=-a_{\mu}^{+} a^{\mu}=a_{i}{ }^{+} a_{i}-1=N-1$, so that each level belongs to a different IR $D(\lambda)$ (discrete series) of $\mathrm{SU}(3,1)$. Eq. (12) may be derived from a Lagrangian.

VI. PROBLEMS OF QUANTIZED FIELDS

The peculiar unconventional properties of infinite component fields with regard to the general conditions of quantum field theory have been widely noted ${ }^{24-29}$. We will confine ourselves here to very brief remarks. Certainly more work is necessary than has been done so far to clarify these problems.

First we note that the results will, in general, depend on whether: a) we regard $\Psi(x)$ simply as a field, or $b$ ) as also 
satisfying a wave equation explicitly or implicitly to define single particle states. In the case a), we may assume all the conventional axioms of field theory (spectrum condition on the states, local commutativity of fields, etc.) except that the fields transform as an infinite dimensional representation of $\mathrm{SL}(2, \mathrm{C})$.

A major difference between finite and infinite representations is that the latter cannot be regarded as a (nonsingular) representation of the complex Lorentz group $\sim \operatorname{SL}(2, C) \otimes S L(2, C)$ because it is infinite-dimensional with respect to the compact imaginary Lorentz transformation. So all the theorems like TCP and spin-statistics, based on such a transformation, are in general expected to break down. (The lack of crossing symmetry in vertex functions was understood in this way earlier.)

This does not mean, however, that these theorems always have to break down. The representations $D(\lambda)$ of $\operatorname{SU}(3,1)$ do have the crossing symmetry (up to a sign) if $|\lambda|$ is an integer. Perhaps this is another attractive feature of $\mathrm{SU}(3,1)$.

In the case b), a great deal depends on the assumed wave equation. Since we have a Lagrangian, it is possible to define the energy-momentum tensor, conserved current vector, etc., and formally introduce the canonical quantization. For a physical theory, the energy density should be positive. Thus, if the wave equation is simple Klein-Gordon type (as in the so-called index-invariant theories), we would need Bose 
statistics, whether the field describes integer or half-integer particles. This requirement also turns out to lead to causal commutation relations. On the other hand, in a Gelfand-Yaglom type equation, Fermi statistics are to be taken for any spin. There may remain some doubt about the validity of canonical quantization and local commutativity. For if the field $\psi(x)$ is supposed to describe a composite system, local commutativity with respect to the "center of mass" coordinates $x$ will not hold. This is probably related to the problem of unphysical spacelike solutions since physical solutions alone would not form a complete set of states, and therefore, would not lead to the local commutation relation required in the canonical quantization scheme.

Although the infinite component fields seem to cause unnecessary relation of general theorems, we emphasize that not everything goes overboard. The conventional TCP and spinstatistics can be preserved if a right group and a right representation are chosen. Besides, they offer a new possibility of deliberately violating, for example, the TCP theorem, microcausality and crossing relation.

\section{SCATTERING PROCESSES}

As a next step in the physical application of infinite component wave equations, we take up the scattering with an external field in Born approximation. We are interested in the intrinsic structure effect such as we have seen in the 
vertex function. For simplicity, we take a scalar external field $\Phi(x)$ coupled to the local current $g(\because(x), Y(x))$, so that the wave equation reads $I_{O} \Psi=g \Phi \psi$. We assume perturbation expansion and obtain for the transition $p+k \rightarrow p^{\prime}+k^{\prime} a$ formal expression

$$
\left(\psi\left(p^{\prime}, n^{\prime}\right), g^{2}\left[\frac{1}{L_{0}(p+k)}+\frac{1}{L_{0}\left(p-k^{1}\right)}\right] \psi(p, n)\right) \equiv M_{1}+M_{2}
$$

corresponding to the usual second order Feynman diagrams. Actually the propagator $\sim 1 / L_{0}$ will involve a summation over all the intermediate states, and this is where interesting properties are expected to emerge.

We emphasize, however, that Feynman perturbation theory and dispersion theory are not necessarily equivalent for infinite component fields, since the usual analyticity assumptions do not hold. Thus we have to compute Eq. (14) directly. For this purpose, it is convenient to use the trick

$$
\frac{1}{L_{O}+i \epsilon}=-i \int_{0}^{\infty} d \tau \exp \left[i L_{O} \tau\right]
$$

(assuming that this gives the right boundary condition). In case $L_{O}$ is linear in the generators of a group $G$, with the coefficients being functions of the momentum, the integrand represents an element of $G$, so that its action on $\psi$ can be determined within, the framework of group theory. Similarly, the initial and final states can be obtained by boosting appropriate rest states. In this way, Eq. (14) is reduced to 


$$
\begin{aligned}
-g^{2} \int_{0}^{\infty} \mathrm{d} \tau\left(\psi\left(\underset{m}{o}, n^{\prime}\right)\right. & \exp \left[-i \vartheta_{p}, K_{p^{\prime}}\right] \exp \left[i L_{o}(p+k) \tau\right] \\
& \left.x \exp \left[i \vartheta_{p} K_{p}\right] \psi(o, n)\right)+\cdots
\end{aligned}
$$

where $\mathrm{K}_{\mathrm{p}}, \mathrm{K}_{\mathrm{p}}^{\prime}$ are the boost generators.

We will treat this in the $\mathrm{SU}(3,1)$ theory with a unitary representation $\lambda<0$ since mathematical manipulation is particularly easy 30,31 . For simplicity, we consider the ground $j=0$ state and equal masses $\mathrm{p}^{2}=\mathrm{p}^{\prime 2}=\mathrm{k}^{2}=\mathrm{k}^{\prime 2}=1$, and work in the C.M. system. The wave equation is generally taken as

$$
L_{O}(p)=-\Gamma_{\mu v} p^{\mu} p^{\nu}\left(p^{2}\right)+g\left(p^{2}\right), \Gamma_{O O}=2(N-\lambda) .
$$

The result is, for the direct channel (s-channel) amplitude $M_{l}$,

$$
\begin{aligned}
& M_{1}=-i g^{2} \int_{0}^{\infty} d \tau\left[\cosh ^{2} \vartheta_{p}-\cos \theta-e^{-2 i \tau s f(s)} \sinh ^{2} \vartheta_{p}\right] e^{\lambda i[g(s)+2 \lambda f(s)]} \\
&=-i g^{2} \int_{0}^{\infty} d \tau\left[\frac{s}{4}-e^{-2 i \tau s f(s)}\left(\frac{t}{2}+\frac{s}{4}-1\right)\right]^{\lambda} e^{i \tau[g(s)+2 \lambda f(s)]} \\
&=\sum_{n=0}^{\infty} \frac{-g^{2}}{2 s f(s)} \oint_{C_{n}} d z z^{\alpha(s)-1}\left[\frac{s}{4}-z^{-1}\left(\frac{t}{2}+\frac{s}{4}-1\right)\right] \\
& \alpha(s)=\frac{g(s)}{2 s f(s)}+\lambda
\end{aligned}
$$

In the last equation, the contour integral is taken along a unit circle round zero repeatedly, as is indicated by the summation sign. This can be summed up to give

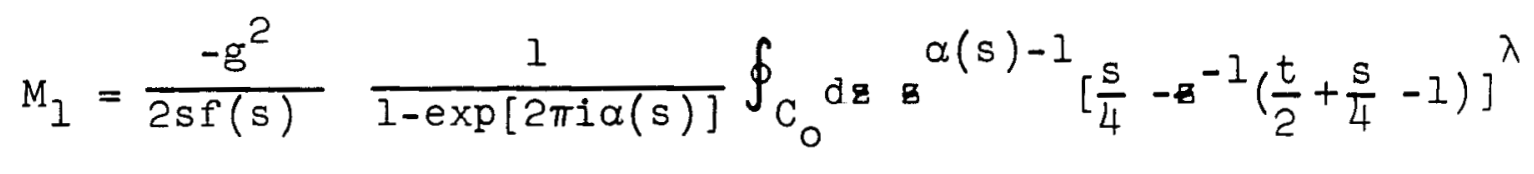

which exhibits the Regge-like resonance factor: there is a 
resonance whenever $a(s)=N=0,1,2,--$ in accordance with the wave equation.

The integrand in Eq. (18) is not singular in $z$ for physical values of $s$ and $t$, but as $s \rightarrow \infty$ the singularity $z_{0}$ approaches the point $z=1$ along the contour, so that an asymptotic expansion may be made. If we assume a straight trajectory $\alpha(s)=s / \mu$, we find.

$$
\mathrm{M}_{1} \sim \frac{\mathrm{g}^{2}}{2 \mathrm{sf}(\mathrm{s})} \frac{\mu}{\mathrm{s}} \cot [\pi \alpha(\mathrm{s}) / \mu] \mathrm{e}^{2 t / \mu}, \quad(\mathrm{s}-\infty)
$$

showing a resonance factor and an optical potential type angular distribution.

The crossed amplitude $\mathrm{M}_{2}$ may be computed in a similar way, but we can easily see the lack of crossing symmetry: it is not the analytic continuation $s-x<0$ of $M_{1}(s, t)$. Moreover, we run into the danger of resonance factors arising from the discrete spacelike solutions unless the wave equation is so chosen that $\alpha(s)<0$ for $s<0$.

The lack of crossing symmetry also fails to make the Regge behavior come out right. The contribution from $M_{2}$ in backward scattering $(x \sim 0)$ looks rather uninteresting as it behaves like $\sim s^{\lambda}$. One may perhaps argue that Regge behavior should not be expected for the present case since it has to do with an external source belonging to a finite multiplet. However, the situation does not seem to change by coupling three infinite component fields through a local vertex. 
VIII. APPLICATION TO HADRON PHYSICS

Let us now briefly discuss the application of our ideas to real hadron physics. It is obvious that the model has to be more complicated than the ones so far considered. But at any. rate, the most interesting and promising approach seems to be the one based on the quark model, assuming the mesons and baryons to belong to definite configurations $q \bar{q}$ and $q q q$ without adding higher configurations. Then the hadronic fields become a product of finite quark spinors and an infinite representation for the orbital motion of the quarks. If only the rotational excitations are considered, the classification of states may be carried out according to the group $G_{0}=S U(3) \times S U(2) \times S O(3)$, or $\mathrm{SU}(6) \times O(3)$ in the well-known manner, and the corresponding G will be SU(3) x SL(2,c) x SO(3,1), or SL(6,c) x SO(3,1). Infinite representations are taken only for the $S O(3,1)$ part. If we include all the internal orbital modes of the quarks, thus making quarks full-fledged quasi-particles, $G_{\circ}$ and $G$ could be made larger. For example, for the mesons $\sim q \bar{q}$, one could use $\mathrm{SO}(4,2)$ or $\mathrm{SU}(3,1)$. However, the harmonic oscillator type representation $D(\lambda)$ of $S U(3,1)$ is not appropriate in this case since it is not self-conjugate. In order to define selfconjugate systems, we have to take $D(\lambda) \pm D(\lambda)^{*}$, thus doubling the space. (Using $S L(4, R)$ instead of $S U(3, I)$ will serve the same purpose.) The number of states becomes essentially the same as in the case of $\mathrm{sO}(4,2)$. 
For the treatment of strong interaction of the hadrons, it is necessary to introduce the coupling of infinite component fields with each other, which we have not discussed yet. If only a (quasi-) local interaction like $C_{n m l} \psi_{n}^{+}(x) \Psi_{m}(x) \Phi_{I}(x)$ is considered, the problem reduces to that of the clebschGordon coefficient for infinite representations. It is known, for example, that there is a unique coupling scheme for $\operatorname{SL}(2, c)$ IR's. In the case of $S U(3,1)$, a simple coupling scheme holds among $D(\lambda), D\left(\lambda^{1}\right)$ and $D\left(\lambda+\lambda^{\prime}\right) *$.

I believe, however, that there is no reason to insist on quasi-local coupling. The best way to avoid unphysical properties may be to stick to a realistic quark model. That is to say, we start from a quark-antiquark wave function $\psi\left(r_{1}, r_{2}\right)$ for the meson, go to the abstract basis $\Psi_{n}(x)$ and assume an equation like (12). For interaction, we can take a form like $\psi\left(r_{1} r_{2}\right) \psi\left(r_{2} r_{3}\right) \psi\left(r_{3} r_{1}\right)$. This will introduce non-local coupling when expressed in terms of the $\psi_{n}(x)$.

Finally, a word about the current algebra. It has been suggested. that infinite dimensional representations of a group may provide non-trivial solutions to the algebra of currents. All indications are, however, that this is not so, and in fact it may be impossible in general.

Let us assume a current of the form $j_{\mu}(x)=\Psi(x) \Gamma_{\mu} \psi(x)$, where $\Gamma_{\mu}$ belongs to the Lie algebra of a group, so we know the commutator $\left[\Gamma_{\mu}, \Gamma_{v}\right]$. But this is not the same thing as the commutator $\left[j_{\mu}(x), j_{U}\left(x^{\prime}\right)\right]$. For the latter depends on the 
commutator of the $\Psi(x)^{\prime}$ s, which must be defined as

$$
\left[\Psi_{n}(x),\left(\bar{\psi}\left(x^{\prime}\right) \Gamma_{0}\right)_{m}\right]_{ \pm}=\delta^{3}\left(x-x^{1}\right) \delta_{n m}
$$

Thus the current commutator amounts to taking

$$
\Gamma_{\mu} \Gamma_{0}^{-1} \Gamma_{U}-\Gamma_{U} \Gamma_{0}^{-1} \Gamma_{\mu}
$$

which in general does not have a simple tensor transformation property required by the current algebra. We can verify this by using IR's of $\mathrm{SL}(2, \mathrm{C})$ for example. For the Majorana representation, the commutator vanishes. 
INPTNITE MIITPPEETS -.- by Y. Nambu

ERPAT I M

Make the following changes or pp. 28-29, REFERENCES:

Item No. 1: Correct spelling from "Gellmann" to "Ge11-iann".

Item No. 21: Omit: "E. Abers, R. E. Worton and C. Fronsdal, preprint."

Insert the following in its place: "G. Cocho, C. Fronsdel, I. I. Grodsky and R. White, preprint."

Item No. 20: Add Pirst inftials to complete autror's name, as follows: "P. T. Matthews". 
1. The basic ideas can be found in a number of papers. E.g., A. O. Barut and A. Böhm, Phys. Rev. 139, B1107 (1965),P. Budini and C. Fronsdal, Phys. Rev. Letters 14, 968 (1965);

Y. Dothan, M. Gellmann and Y. Ne'eman, Phys. Letters 17 , 148 (1965); N. Mukunda, L. O'Rafaertaigh and E. C. G. Sudarshan, Phys. Rev. Letters 15, 1041 (1965); T. Takabayashi, Progr. Theor. Phys. Suppl. (Kyoto), Extra Number, 339 (1965); A. O. Barut, in Non-Compact Groups in Particle Physics, edited by $\mathrm{Y}$. Chow (W. A. Benjamin and Company, Inc., New York, 1966).

2. A. O. Barut and A. Bohm, Ref. I.

3. N. Mukunda, L. O'Rafaertaigh and E. C. G. Sudarshan, Ref. 1.

4. Y. Ne'eman, Algebraic Theory of Particle Physics, W. A. Benjamin, Inc., Publ., New York, 19607.

5. T. Takabayashi, Ref. 1.

6. H. Yukawa, Phys. Rev. 77, 219 (1955); 91, 415 (1953).

7. E. Majorana, Nuovo Cimento 9, 335 (1932).

8. - -

9. - -

10. C. Fronsdal, preprint.

11. I. M. Gelfand and A. M. Yaglom, Zh. Exper. i Teoret. Fiz. 18, 703 (1948). M. A. Naimark, Linear Representations of the Lorentz Group (English transl., Pergamon Press, Inc., London, 1964 ).

12. V. Bargmann, Ann. Math. 48, 568 (47).

13. W. Rdhl, preprint.

14. F. Gulrsey, Proc. Coral Gables Conference on Symmetry Principles at High Energy, 1967. 
15. G. Feinberg, preprint.

16. Y. Nambu, Dalhousie Summer Schsol Lectures, Univ. Delhi, 1967.

17. A. O. Barut and H. Kleinert, Proc. NATO Advanced Study Inst., Istanbul, 1966.

18. Y. Nambu, Progr. Theoret. Phys. Suppl. Nos. 37 and 38 , 368 (1966); Phys. Rev. 160;1171(1967).

19. C. Fronsdal, Phys. Rev. 156, 16.6. (1967).

20. Y. Nambu, Proc. Coral Gables Conference on Symmetry Principles at High Energy, 1967, and Ref. 18.

21. S. P. Rosen, unpublished. E. Abers, R. E. Norton and C. Fronsdal, preprint.

22. T. Takabayashi, preprint.

23. M. Gell-Mann and R. P. Feynman, private communication.

24. I. M. Gelfand and A. M. Yaglom, Zh. Exper. i. Teoret. Fiz. 18, 1096 (1948).

25. B. Zumino, Proc. Seminars on Unified Theories of Elementary Particles (Munich) 1965.

26. G. Feldman and Matthews, Phys. Rev. 154, 1241(1967).

27. C. Fronsdal, Phys. Rev. 156, 1653 (1967).

28. E. Abers, I. T. Gro dsky and R. E. Norton, Phys. Rev. 159, 1222 (1967).

29. ITodorov, preprint.

30. G. Cocho, preprint.

31. Y. Nambu, to be published. 